\title{
Vanishing lung syndrome with community-acquired pneumonia and infection of bullae
}

\begin{abstract}
We present a case of a 36-year old male who was a long-term smoker and was found to have giant bullous emphysema on chest imaging as an accidental finding. At the time, when his first chest CT was obtained, he was asymptomatic and was recommended to consult a pulmonologist but was lost to follow-up for a year until he presented to the emergency department with fever, dyspnea, and chest pain. He was admitted to a pulmonology department. Chest CT was performed and it revealed infected bullae containing air-fluid levels as a complication of community-acquired pneumonia. After successful antibacterial treatment, the patient was discharged and recommended to consult with a thoracic surgeon. A few months later, he had video-assisted thoracoscopic surgery and left upper lobectomy as part of definitive treatment.
\end{abstract}

Key words: giant bullous emphysema, vanishing lung syndrome, infected bullae, pneumonia

Adv Respir Med. 2021; 89: 451-455

\section{Introduction}

Giant bullous emphysema (GBE), also known as vanishing lung disease or type I bullous disease, is defined by giant bullae in one or both upper lobes occupying at least one-third of the hemithorax and compressing the normal surrounding parenchyma [1]. It is a progressive disease in which the affected regions of the lung do not participate in gas exchange often leading to dyspnea, hypoxia, chest tightness, and spontaneous pneumothorax. Vanishing lung disease can often be asymptomatic, especially in younger patients, and manifests only when complications occur - most commonly infection of bullae or pneumothorax. The development of GBE is associated with smoking, inhaled drug abuse, alpha-1 antitrypsin deficiency, and connective tissue disorders.

\section{Material and methods}

A 36 year-old patient was admitted to the emergency department complaining of left-sided chest pain worsened by coughing and deep in- spiration, cough with yellow-greenish discharge, non-foul smelling sputum, and dyspnea of a few days duration. Two days before hospitalization, he developed chills and fever up to $39^{\circ} \mathrm{C}$. Upon admission, he had fever of $38.5^{\circ} \mathrm{C}$, respiratory rate of $24 \times / \mathrm{min}$, and $\mathrm{SpO}_{2}$ at $93 \%$ on room air. Respiratory examination revealed crackles and wheezing on the left side. The examination of other systems was unremarkable.

Laboratory examination revealed neutrophilic leukocytosis (leukocytes $18.8 \times 10^{9} / \mathrm{L}$ ) and increased CRP (211.3 mg/L). Other results were within reference ranges. Chest X-ray was performed and revealed consolidations on the left side with air-fluid levels and blunting of the left costophrenic angle (Figure 1). There was suspicion of possible empyema with free air in the pleural space.

For further investigations and treatment, the patient was admitted to the pulmonology department. Before initiating therapy with amoxicillin/clavulanic acid, sputum and blood cultures were drawn. A chest CT scan was performed and revealed centrilobular and paraseptal emphysema predominantly in apical parts of the lungs and 


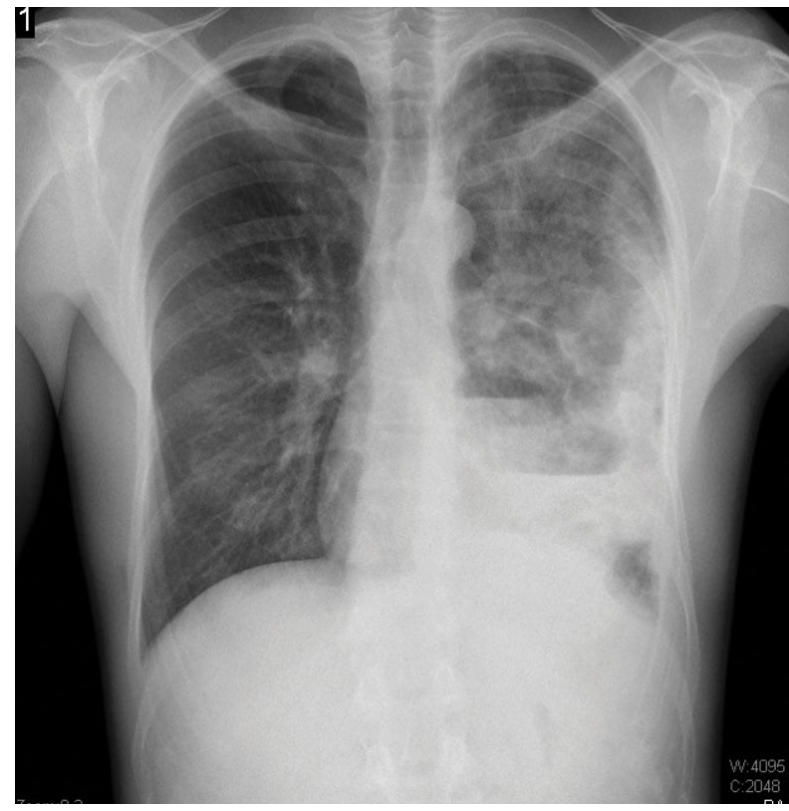

Figure 1. Chest $X$-ray PA revealing consolidations of the left lung with air-fluid levels and blunting of left costophrenic angle a bulla in the left apex $(\sim 16 \times 8.6 \times 7.3 \mathrm{~cm})$ with air-fluid levels. Another perihilar cavity with air-fluid levels was seen between the $6^{\text {th }}$ and $8^{\text {th }}$ segment together with smaller bullae in the $2^{\text {nd }}$ and $9^{\text {th }}$ segment. Free fluid in the left pleural space was noted up to $3.6 \mathrm{~cm}$ (Figure 2).

Even though the patient had purulent sputum, the sputum culture came back unspecific revealing only normal upper respiratory flora. Blood cultures were negative. Treatment with amoxicillin/clavulanic acid was ineffective and patient still had a fever up to $39^{\circ} \mathrm{C}$. Inflammatory markers were increasing which prompted a change in antibacterial treatment to piperacillin/tazobactam. On this regiment, the patient's overall condition rapidly improved and he became afebrile in 4 days. One week later, his inflammatory markers markedly decreased (leukocytes - $15.29 \times$ $10^{9} / \mathrm{L}$, CRP $\left.-17.4 \mathrm{mg} / \mathrm{L}\right)$. A follow-up CT scan 2 weeks after initiation of piperacillin/tazobactam revealed improvement in pneumatization of the
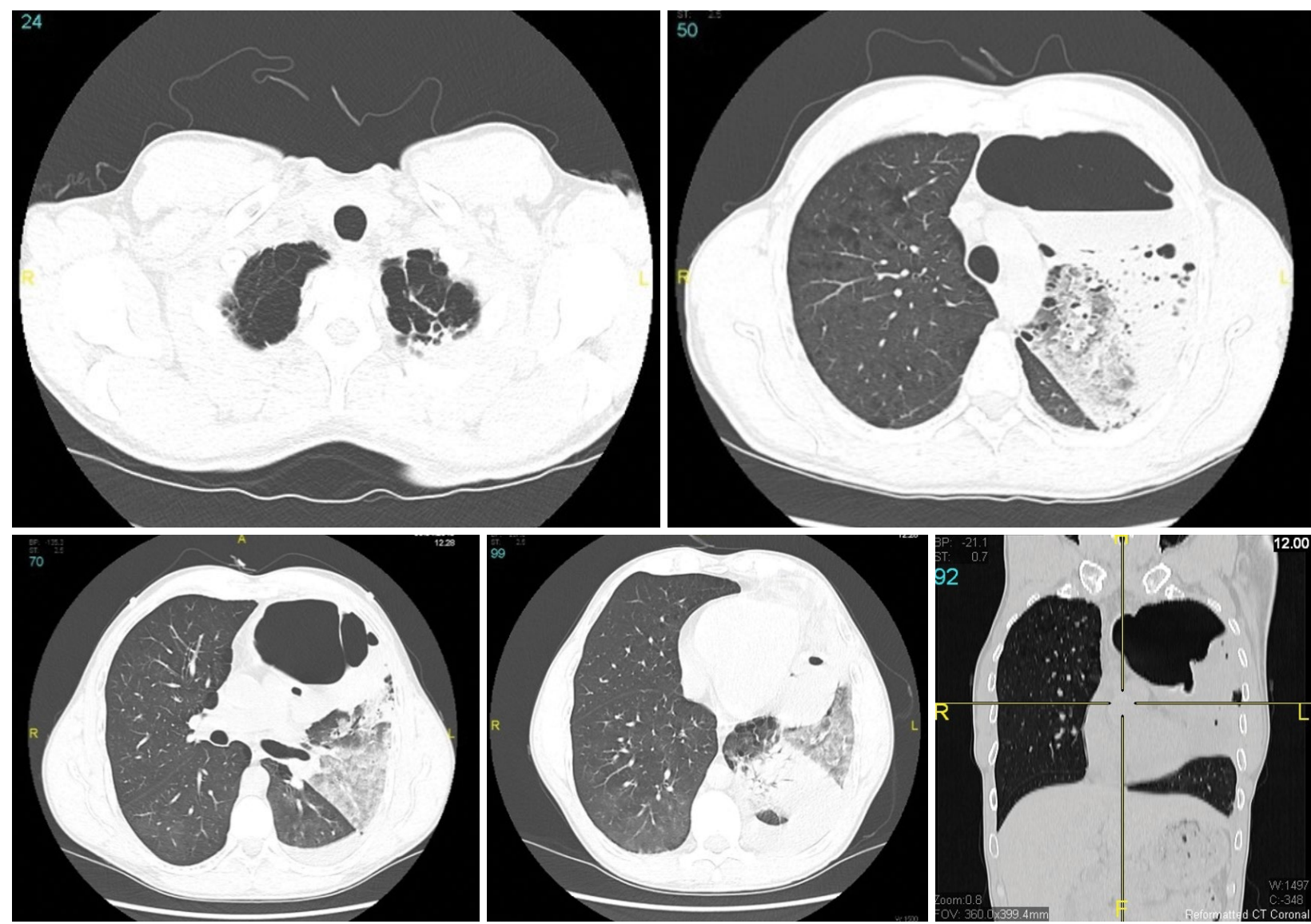

Figure 2. Chest computed tomography in coronal and axillar plane revealing bulla on the left side with air fluid levels, adjacent consolidation of the lung, and pleural effusion 

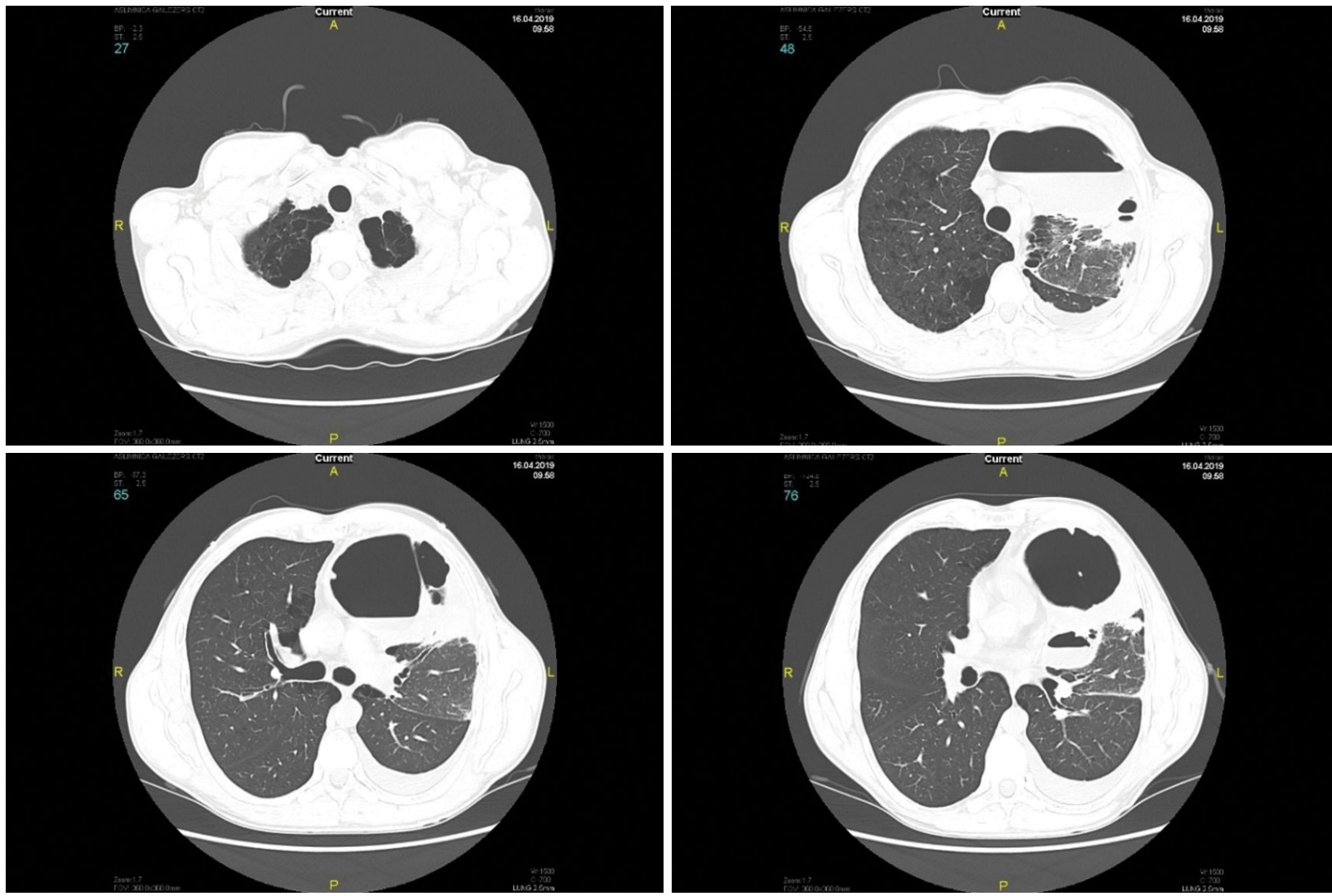

Figure 3. Chest computed tomography 2 weeks after initiation of antibacterial treatment with piperacillin/tazobactam. Air-fluid level in the bullae still present, but marked improvement in pneumatization of the lung is seen

left lung, but the bullae were still filled with fluid (Figure 3). The patient was referred to a thoracic surgeon and uniportal video-assisted thoracoscopy (VATS) with left upper lobectomy was indicated. The patient didn't agree to any further treatment in the hospital and, against medical advice, was discharged with recommendations to consult a thoracic surgeon. Two months later, the patient was seen by a surgeon and was admitted to the thoracic surgery department for VATS. After a successful postsurgical period, the patient was lost for further follow-up as was expected considering his low adherence to treatment and recommendations.

During the time of hospitalization and after additional questioning of the patient, it was determined that he was a long-term smoker who had been smoking approximately 30 cigarettes a day since the age of 6 ( 45 pack years). As well, he unwillingly revealed to have often smoked marijuana as well. At the time of hospitalization, he had switched to electronic cigarettes, but was not able to quit smoking completely. He admitted that a year prior to his present hospitalization episode, he was hospitalized at another hospital with acute pancreatitis. There, a CT was performed that accidentally revealed giant bullous emphysema. The bullae had a "mass effect", protruding and restricting left lung upper segments and moving the heart shadow to the right (Figure 4).

After marked improvement of the patient's overall condition, full pulmonary function tests were performed which revealed air trapping (defined as an increase in RV and FRC) and decreased diffusing capacity - FEV1/FVC $80.30 \%$, FEV1 82.5\%, TLC 102.7\%, RV 164\%, FRC 175\%, DLCO 65.7\%. $\alpha 1$-antitrypsin plasma levels were within the normal range $(1.8 \mathrm{~g} / \mathrm{L}$; reference values $0.92-2.0 \mathrm{~g} / \mathrm{L})$.

\section{Discussion}

Giant bullous emphysema (GBE), also referred to as vanishing lung syndrome, is a rare condition that typically presents in young male smokers or $\alpha_{1}$-antitrypsin deficient (AATD) patients. Bullae are formed by the destruction of interalveolar walls due to chronic or acute stretch injury with increased intraalveolar pressure [2]. On chest radiograph, a bulla has to take no less 

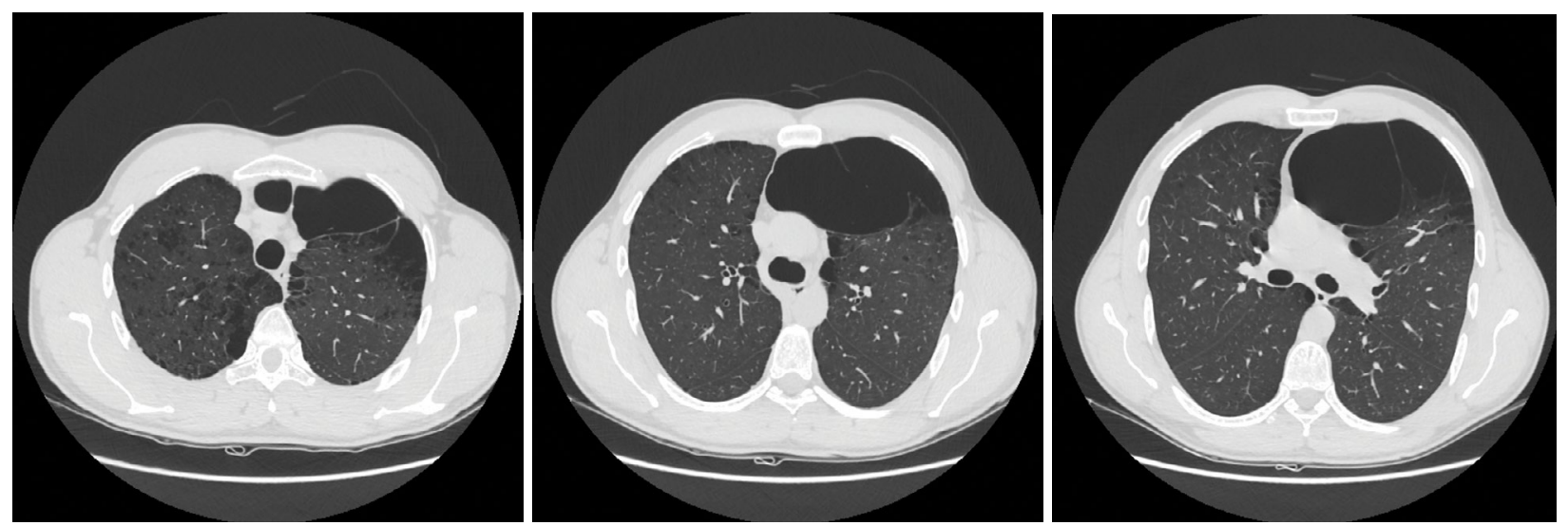

Figure 4. Chest CT performed one year prior, revealing giant bullous emphysema

than $30 \%$ of the hemithorax to be considered a giant bulla. Commonly, such patients present with progressive dyspnea due to the bulla compressing surrounding lung tissue and disturbing optimal gas exchange, but sometimes these patients can be asymptomatic and be unaware of the existing pathology [3].

The exact prevalence of vanishing lung syndrome remains unknown as only a small number of case reports and series have been reported. The specific cause of the pathology is obscure, but male predisposition and smoking (both cigarette and marijuana abuse) have been observed to be risk factors [4]. Similar to how only about $30 \%$ of smokers develop emphysema (mostly panacinar), it is reasonable to assume that yet to be identified triggers and predisposing factors (acquired or inherited) lead to the development of GBE in the same population. On the other hand, the pathogenesis of bulla formation is similar and is due to an imbalance of proteases/antiproteases and oxidants/antioxidants in the lung. This disequilibrium causes a chain reaction at a cellular level leading to the eventual destruction of alveolar walls resulting in permanent and abnormal enlargement of distal airspaces [5]. Bullae developing in emphysema are air-filled, thin-walled cavities larger than $1 \mathrm{~cm}$ in diameter leading to poor gas exchange due to reduced total alveolar surface area and fibrosis of the alveolar membrane [6].

In patients with established GBE, pulmonary function tests and $\alpha_{1}$-antitrypsin serum level measurements must be performed. In the reported patient, AATD was initially highly suspected. However, as plasma levels were normal, no further genetic studies were performed because it is not reimbursed by the government in our country. There is no precise data of $\alpha_{1}$-antitrypsin defi- ciency prevalence in Latvia. The European data is scarce - it varies from 1 in 1,368 in Denmark to 1 in 58,319 in Poland [7].

The natural history of vanishing lung syndrome is reported to be a progressive one, but the rate of it is highly variable [8]. Bullae can enlarge, compressing healthy lung parenchyma and interfering with normal respiratory mechanics and gas exchange (increase in dead space fraction). Lung compliance decreases and work of breathing increases [5]. Usually, in patients with emphysema, we would expect to have a typical obstructive pattern in spirometry. However, as demonstrated by this patient, it is not always the case in GBE. This is also why the use of spirometry as a follow-up tool is questionable. Regarding follow-up, the most important issue is to monitor the patient closely for worsening of symptoms and for the development of possible complications.

In patients with GBE, important steps in treatment include smoking cessation and adherence to vaccination schedules. It is assumed that in asymptomatic patients with GBE, a reasonable first-line approach is the use of bronchodilators. However, as patients become more symptomatic or develop complications, especially pneumothorax, surgical intervention is required.

The treatment of choice in GBE is bullectomy. Available data suggests that elective bullectomy has good results. In a 5-year follow-up study of 41 patients with giant bullous emphysema who had undergone elective bullectomy, improvements in symptoms and lung function (significant improvements in lung volumes, FEV1, and the FEV1/ FVC ratio over baseline) were noted. As well, bullae did not reappear and residual bullae did not become enlarged at the site of the bullectomy. During follow-up, the dyspnea score was reduced significantly soon after bullectomy 
and remained so even up to the fourth year of follow-up. The bullectomy approach is associated with a decreased risk of infection and pneumotho$\operatorname{rax}[9]$. As of now, there is no consensus available on the ideal timing for surgery or even follow-up of GBE patients.

The reported patient was asymptomatic and was unaware of the existing pathology until hospitalization due to acute pancreatitis and accidental CT findings of bullous emphysema. At that moment, his pulmonary function tests were better than expected - the deviations seen in this patient's case were increased RV and FRC (above $120 \%$ ) with normal TLC levels, as well as decrease in DLCO due to bullae leading to a decrease in surface area where gas exchange takes place. As a result, the consulting pulmonologist prescribed treatment with bronchodilators. However, the patient was not adherent to the treatment protocol as the bullous changes were not causing any symptoms and did not interfere with his daily activities. One year later, he presented with acute worsening of the symptoms and community-acquired pneumonia with infection of the bullae which prompted a lengthy stay in the hospital and surgical intervention. This case shows that even though the disease is asymptomatic, follow-up is important as pathology can lead to serious complications such as infection or rupture of bullae, total pneumothorax, or infection-linked life-threatening conditions.

\section{Conflict of interest}

None declared.

\section{References:}

1. Roberts L, Putman CE, Chen JT. Vanishing lung syndrome: upper lobe bullous pneumopathy. Rev Interam Radiol. 1987; 12: 249-255.

2. Klingman RR, Angelillo VA, DeMeester TR. Cystic and bullous lung disease. Ann Thorac Surg. 1991; 52(3): 576-580, doi: 10.1016/0003-4975(91)90939-n, indexed in Pubmed: 1898156.

3. Deslauriers J, Leblanc P. Management of bullous disease. Chest Surg Clin N Am. 1994; 4(3): 539-559, indexed in Pubmed: 7953483.

4. Hii SW, Tam JDC, Thompson BR, et al. Bullous lung disease due to marijuana. Respirology. 2008; 13(1): 122-127, doi: 10.1111/j.1440-1843.2007.01186.x, indexed in Pubmed: 18197922.

5. Stern EJ, Frank MS. CT of the lung in patients with pulmonary emphysema: diagnosis, quantification, and correlation with pathologic and physiologic findings. AJR Am J Roentgenol. 1994; 162(4): 791-798, doi: 10.2214/ajr.162.4.8140992, indexed in Pubmed: 8140992.

6. Im Y, Farooqi S, Mora A. Vanishing lung syndrome. Proc (Bayl Univ Med Cent). 2016; 29(4): 399-401, doi: 10.1080/08998280.2016.11929486, indexed in Pubmed: 27695175.

7. Blanco I, de Serres FJ, Fernandez-Bustillo E, et al. Estimated numbers and prevalence of $\mathrm{PI}^{\star} \mathrm{S}$ and $\mathrm{PI}^{\star} \mathrm{Z}$ alleles of alpha1-antitrypsin deficiency in European countries. Eur Respir J. 2006; 27(1): 77-84, doi: 10.1183/09031936.06.00062305, indexed in Pubmed: 16387939.

8. Tay CK, Ng YL. A breath from Houdini - a case of giant bullous emphysema. Respir Med Case Rep. 2014; 14: 30-33, doi: 10.1016/j.rmcr.2014.12.003, indexed in Pubmed: 26029573.

9. Palla A, Desideri M, Rossi G, et al. Elective surgery for giant bullous emphysema: a 5-year clinical and functional follow-up. Chest. 2005; 128(4): 2043-2050, doi: 10.1378/ chest.128.4.2043, indexed in Pubmed: 16236853. 